\title{
Strain Gradient Polycrystal Plasticity for Micro-Forming
}

\author{
Tuncay Yalçinkaya ${ }^{1, a)}$, Igor Simonovski ${ }^{2, b)}$ and İzzet Özdemir ${ }^{3, c)}$ \\ ${ }^{1}$ Department of Aerospace Engineering, Middle East Technical University, Ankara 06800, Turkey. \\ ${ }^{2}$ European Commission, Joint Research Centre, Institute for Energy and Transport P.O. Box 2, NL-1755 ZG Petten, \\ The Netherlands. \\ ${ }^{3}$ Department of Civil Engineering, Izmir Institute of Technology, Urla, Izmir 35430, TURKEY \\ a)Corresponding author: yalcinka@metu.edu.tr \\ b)Igor.Simonovski@ec.europa.eu \\ c)izzetozdemir@iyte.edu.tr
}

\begin{abstract}
The developments in the micro-device industry has produced a substantial demand for the miniaturized metallic components with ultra-thin sheet materials that have thickness dimensions on the order of 50-500 $\mu \mathrm{m}$ which are produced through micro-forming processes. It is essential to have predictive tools to simulate the constitutive behavior of the materials at this length scale taking into account the physical and statistical size effect. Recent studies have shown that on the scale of several micrometers and below, crystalline materials behave differently from their bulk equivalent due to micro-structural effects (e.g. grain size, lattice defects and impurities), gradient effects (e.g. lattice curvature due to a non-uniform deformation field) and surface constraints (e.g. hard coatings or free interfaces). These effects could lead to stronger or weaker material response depending on the size and unique micro-structural features of the material. In this paper a plastic slip based strain gradient crystal plasticity model is used to address the effect of microstructural features (e.g. grain size, orientation and the number of grains) on the macroscopic constitutive response and the local behavior of polycrystalline materials.
\end{abstract}

\section{INTRODUCTION}

The ongoing miniaturization in the micro-electronics and micro-devices industry is increasing the need for predictive tools that could link the information at the micro scale to the observations at the engineering scale. Traditional constitutive models based on continuum mechanics are not able to capture the size dependent plastic behavior of materials when the specimen size goes to micron levels. In particular during the forming processes at this length scale two types of size effects have been observed, i.e. the physical and the statistical size effects. The physical size effects are due to the underlying physical mechanisms such as dislocation-grain boundary interaction and heterogeneous plastic strain evolution influencing the hardening behavior of the component as it is miniaturized. On the other hand statistical size effect comes from the fact that when the size of the specimen decreases, the number of grains is restricted and the behavior of the individual grains become more decisive on the macroscopic response. The current paper studies the physical size effect in polycrystalline metals through recently developed plastic slip based strain gradient crystal plasticity framework (see [1], [2]) which is used with Voronoi tessellation to generate the aggregate model. Current study addresses physical size effect simulations at 2D in a plane strain context. In order to illustrate this effect more clearly the hardening is based solely on gradient terms.

\section{MODEL DESCRIPTION}

Voronoi tessellation [3] is used to obtain a basic geometry of the polycrystalline aggregate. In our case the aggregate contains 212 grains (see e.g. Fig. 1). Initial average grain size is set to $D_{\text {avg }}=50 \mu \mathrm{m}$. The aggregates are then scaled to obtain average grain sizes from 0.5 to $200 \mu \mathrm{m}$. This compares well with the average grain sizes of AISI 304 stainless steels (1 to $47 \mu \mathrm{m},[4])$ and AISI 316L (13 to $107 \mu \mathrm{m}[5])$. 


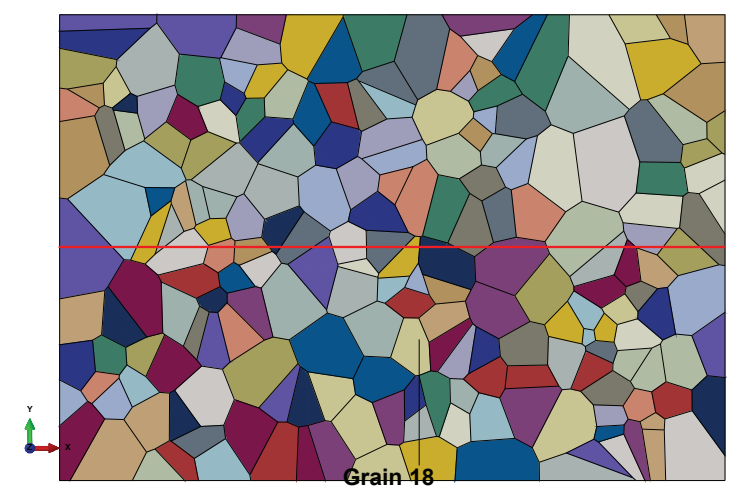

FIGURE 1. 212 grain model. The red line indicates the path along which $\epsilon$ and $\sigma$ values are displayed in Fig. 3 .

\section{Loads and boundary conditions}

Displacement load is applied to the left and right edge in the global $-X$ direction (left edge) and the global $+X$ direction (right edge), resulting in macroscopic $\left\langle\epsilon_{11}\right\rangle=10 \%$. The symbol \langle\rangle represents the Macaulay bracket, indicating a macroscopically averaged value. Rigid body movement is prevented by fixing the bottom left and bottom right nodes of the model in global Y direction. If one imagines that the aggregate is embedded in a larger piece of material, the applied boundary condition lies between the two bordering cases of constraints, imposed to the aggregate by the surrounding material: a) stresses or b) displacements imposed on the aggregate boundaries. The latter condition results in straight edges. Plastic slip at the boundaries of the model is not constrained.

\section{Material parameters}

Material parameters are taken directly from the literature (see [2]) and are used to demonstrate the strain gradient effects in the polycrstalline aggregates. They are not directly related to any engineering materials. Table 1 lists the used strain gradient crystal plasticity material properties. Crystallographic orientations of grains are randomly distributed $\left(0-360^{\circ}\right)$ using uniform probability distribution. Three slip systems are considered in each grain.

TABLE 1. Material properties of the strain gradient single crystal plasticity model of [1], [2].

\begin{tabular}{|c|c|c|c|c|c|c|c|c|}
\hline $\begin{array}{c}\text { Young } \\
\text { modulus } \\
\mathrm{E}[\mathrm{MPa}]\end{array}$ & $\begin{array}{c}\text { Poisson } \\
\text { ratio } \\
v[/]\end{array}$ & $\begin{array}{c}\text { Reference } \\
\text { slip rate } \\
\dot{\gamma}_{0}\left[\mathrm{~s}^{-1}\right]\end{array}$ & $\begin{array}{c}\text { Slip } \\
\text { resistance } \\
\mathrm{s}[\mathrm{MPa}]\end{array}$ & $\begin{array}{c}\text { Angle1 } \\
\left.{ }^{\circ}\right] \\
\end{array}$ & $\begin{array}{c}\text { Angle2 } \\
\left.{ }^{\circ}\right] \\
\end{array}$ & $\begin{array}{c}\text { Angle3 } \\
\left.{ }^{\circ}\right] \\
\end{array}$ & $\begin{array}{c}\text { Material } \\
\text { length scale } \\
R[\mu \mathrm{m}] \\
\end{array}$ & $\begin{array}{c}\text { Thickness } \\
\text { [mm] }\end{array}$ \\
\hline 210000.0 & 0.33 & 0.15 & 20.0 & 120.0 & 60.0 & 45.0 & $0.0,1,5,7.5,10$ & 1.0 \\
\hline
\end{tabular}

\section{RESULTS}

Fig. 2 shows the effect of the material length scale parameter $R$ on the macroscopic stress-strain response. The $R$ parameter here is basically the radius of a circle within which dislocations are assumed to contribute to the internal stress field (see [6]). In these types of models the internal length scale could be related to different microstructural features and the value would vary, e.g. [7] relates it to dislocation spacing, [8] to dislocation source distance and [9] to grain size. In this work we relate $R$ to a certain percentage of the grain size to study its influence.

First of all when $R$ is set to 0 the model reduces to classical crystal plasticity and identical response curves are obtained for the cases with different average grain size. Fig. 2 shows the effect of $R=5 \mu \mathrm{m}$ (left) and $R=10 \mu \mathrm{m}$ on the macroscopic stress-strain response. Since models with different average grain sizes are used, the resulting $R / D_{\text {avg }}$ ratios range from $2.5 \%$ to $100 \%$. The latter value is of course significantly too high but it is used here to show the trends in the macroscopic responses. On can observe that: a) higher $R / D_{\text {avg }}$ ratio results in a stiffer response and $\mathrm{b}$ ) 

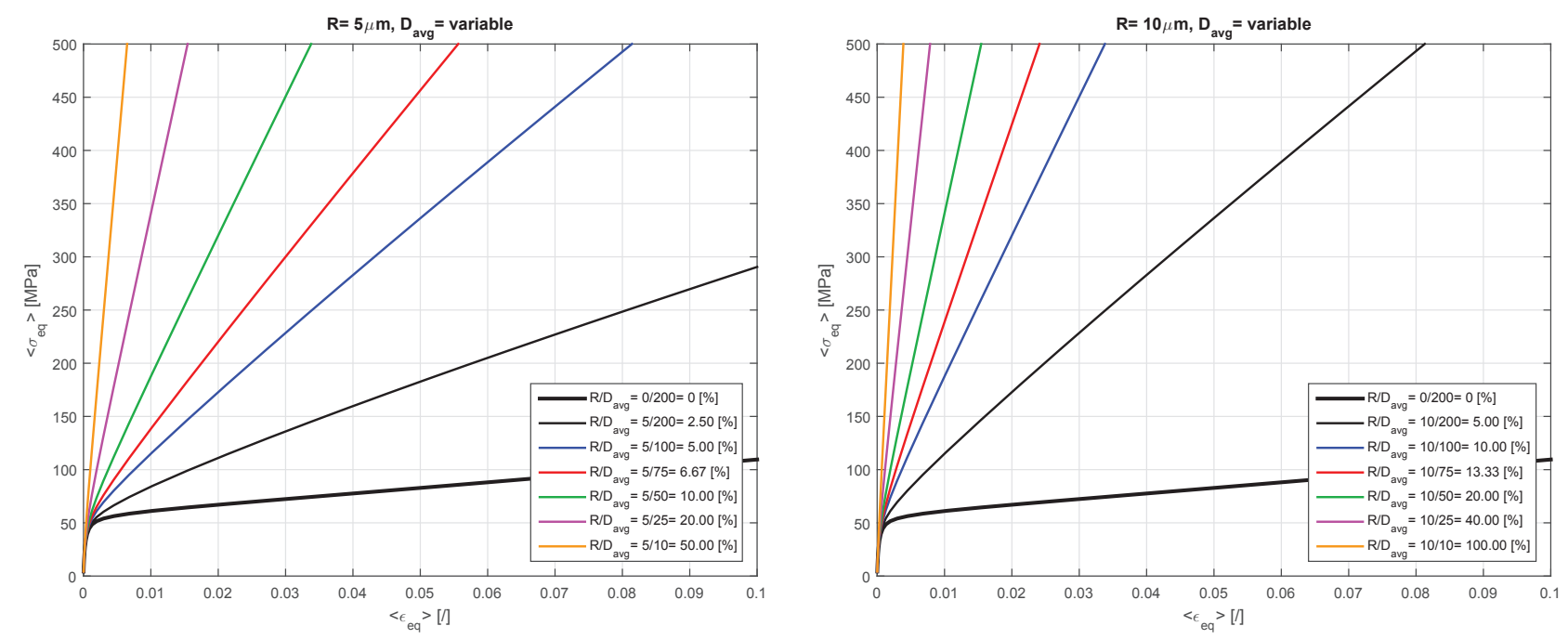

FIGURE 2. The effect of the material length scale parameter $R$ on the macroscopic stress-strain curves.
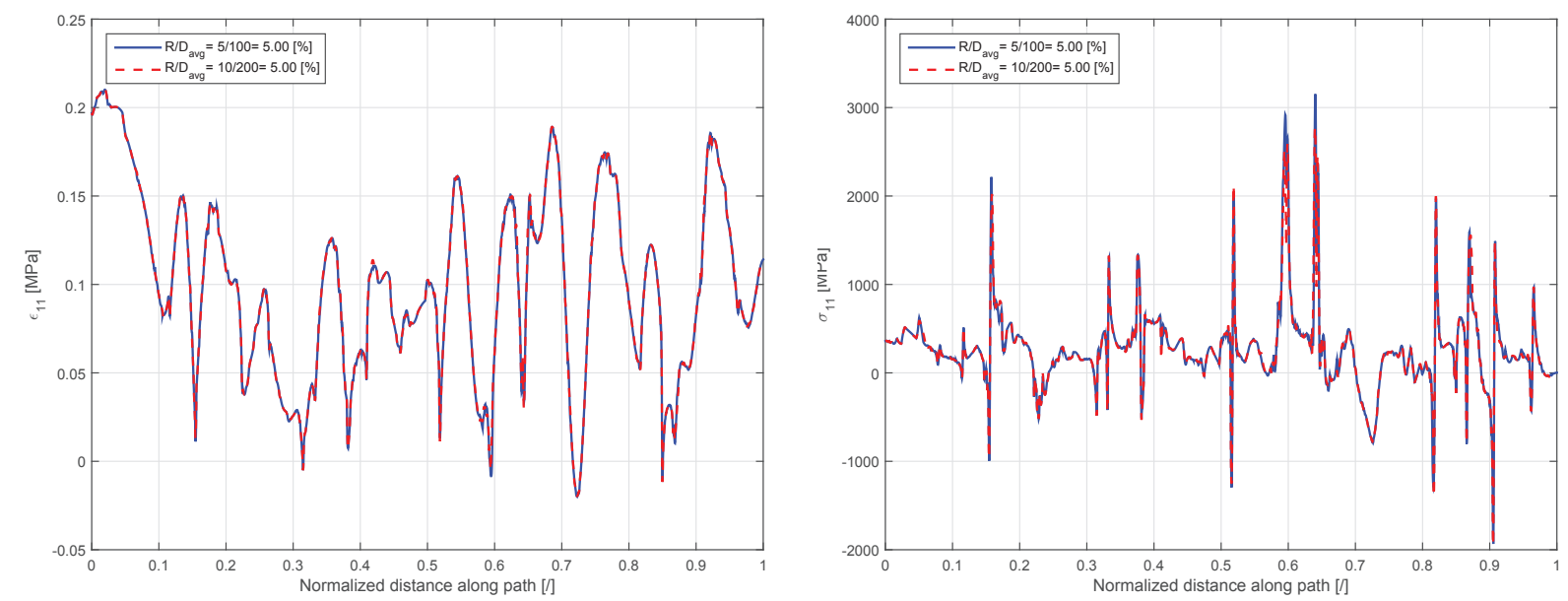

FIGURE 3. Variation of $\epsilon_{11}$ and $\sigma_{11}$ along the horizontal path (Fig. 1) for $R / D_{\text {avg }}=5 \% . R=[2.5 \mu \mathrm{m}, 5 \mu \mathrm{m}], D_{\text {avg }}=[50 \mu \mathrm{m}, 100 \mu \mathrm{m}]$.

the same macroscopic response is observed as long as the ratio $R / D_{a v g}$ remains the same. The latter is not only true for the macroscopic response but for the local response as well. Fig. 3 shows the $\epsilon_{11}$ and $\sigma_{11}$ values along the model's horizontal path (red line in Fig. 1) for $R / D_{a v g}=5 \%: 5 \mu \mathrm{m} / 100$ and $10 \mu \mathrm{m} / 200 \mu \mathrm{m}$. Fig. 4 shows the $\epsilon_{11}$ and $\sigma_{\text {Mises }}$ distributions within grain 18. Identical results are obtained for two different $R$ values, keeping in mind that the ratio $R / D_{\text {avg }}$ is the same in both cases.

Average grain size has a significant effect on the yield strength of a material. It directly affects the number of grain boundaries in a material. Since grain boundaries impede the movement of dislocations a material with larger number of grain boundaries has a higher yield stress. This is a well-known Hall-Petch relation, $\sigma=\sigma_{o}+K_{p} / \sqrt{D}$ (see [10], [11], [12], where $\sigma$ stands for the yield stress, $\sigma_{o}$ for the lattice frictional stress, $K_{p}$ is a material constant and $D$ stands for the grain size. This equation is valid up to about grain size of $10 \mathrm{~nm}$ at which point the grains are too small to fit more than two dislocations. Consequently, decreasing the grain size below $10 \mathrm{~nm}$ results in lower yield strength.

In this context, Figure 5 shows the effect of the material length scale parameter $R$ on the $0.2 \%$ proof stress. In the left part of the figure one can see that the proof stress increases with decreased average grain size, mostly in the 

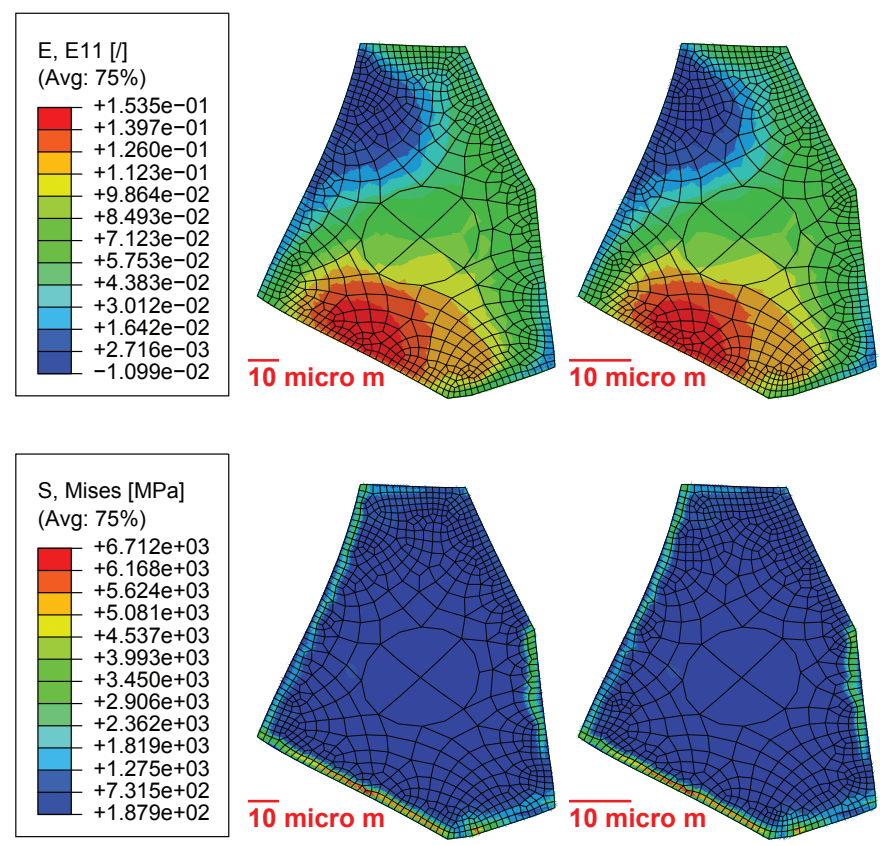

FIGURE 4. $\epsilon_{11}$ and $\sigma_{\text {Mises }}$ in grain 18 for $R / D_{\text {avg }}$ ratios of $5 \mu \mathrm{m} / 100 \mu \mathrm{m}=5 \%$ (left) and $10 \mu \mathrm{m} / 200 \mu \mathrm{m}=5 \%$ (right). Note the difference in the grain size.
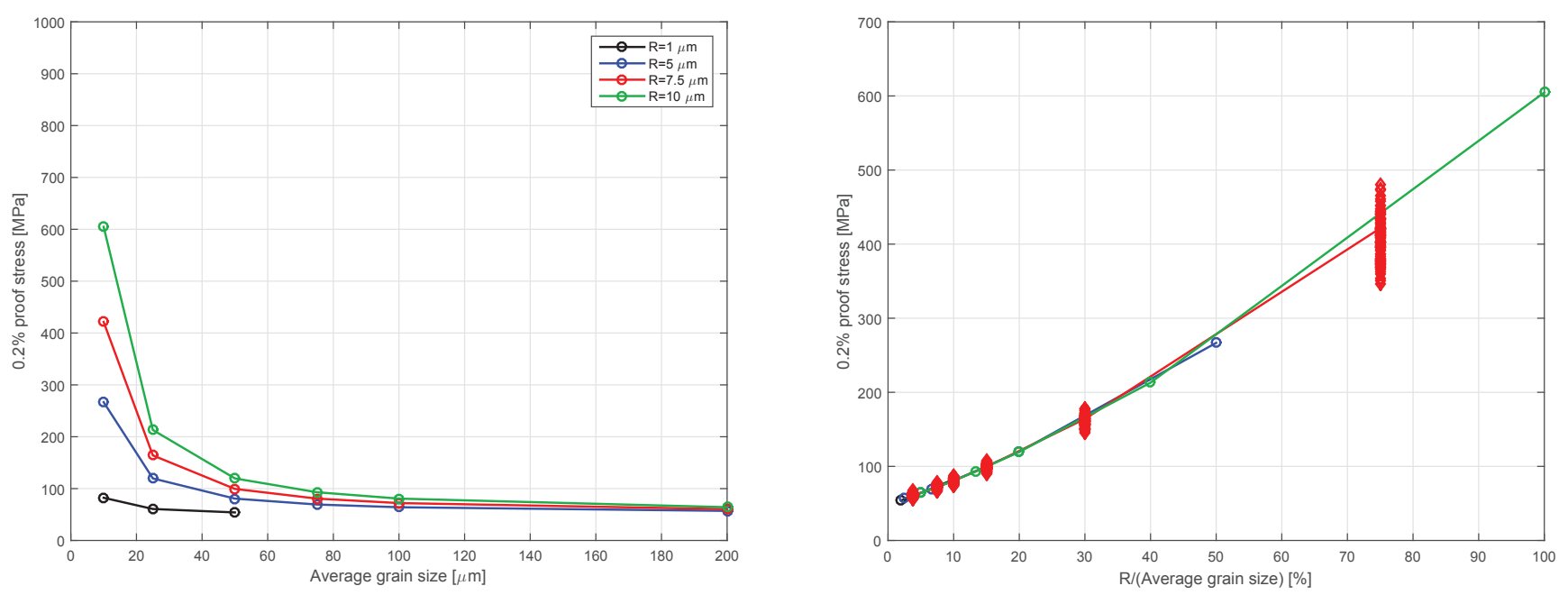

FIGURE 5. The effect of the material length scale parameter $R$ on the $0.2 \%$ proof stress.

region where average grain size are between 10 and $50 \mu \mathrm{m}$. However, this part of the figure is obtained by keeping the $R$ parameter constant while reducing the average grain size. Consequently, the $R / D_{\text {avg }}$ increases which in itself results in stiffer response, as shown in Figure 2. The right part of the figure displays the same data but plotted against the $R / D_{\text {avg. }}$. In this case practically all the data falls onto the same line. The increase in the proof stress is evident from $57.2 \mathrm{MPa}$ at $R / D_{\text {avg }}=2.5 \%, 213 \mathrm{MPa}$ at $R / D_{\text {avg }}=40 \%$ to $266.7 \mathrm{MPa}$ at $R / D_{\text {avg }}=50 \%$. For the latter case the $R / D_{\text {avg }}$ is obviously already beyond the physical range as $R$ should not be larger than half the average grain size. However, the results do show that the larger the radius of a circle within which dislocations contribute to the internal stress field (i.e. $R$ ), the larger the proof stress is. On the other hand the larger the $R$ the more the stresses are averaged within 
individual grains. Different sets of grain crystallographic orientations introduce scatter in the results, indicated by red diamonds. 100 different sets of crystallographic orientations were used at given $R / D_{\text {avg }}$ ratio.

Figure 6 shows the equivalent stress and strain distributions in grain 18 of the aggregate. In small ranges of $R / D_{\text {avg }}$ ratio, i.e. $2.5 \%$ and $5 \%$ high stress concentrations at the grain boundaries and high strain values around the center of the grain are observed. As the material length scale parameter $R$ is increased, larger internal stresses are obtained penalizing high plastic slip gradients and resulting in more spread GNDs and diluted stress and strain concentrations.
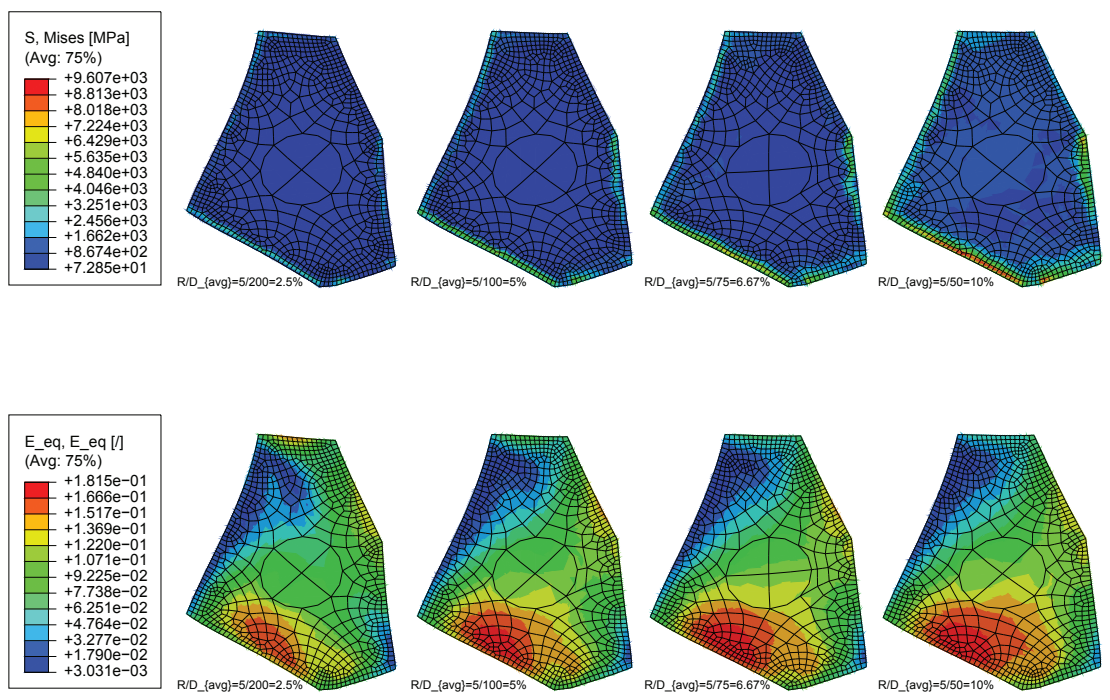

FIGURE 6. Variation of $\sigma_{\text {Mises }}$ and $\epsilon_{e q}$ within grain 18 for different $R / D_{\text {avg }}$ values.

An important parameter in strain gradient crystal plasticity simulations is the finite element size around the high strain/stress gradient areas. Within these areas the element size should be smaller than $R$. Preferably the element size should be $R / 2$ or smaller. This is the case with all the models and results displayed so far. Fig. 7 shows the effect of the element size on the local stress field within grain 8 of an aggregate containing 14 grains in total. $R$ value of $0.5 \mu \mathrm{m}$ is used and the element size at the grain boundary is progressively decreased from 12.5 to $0.5 \mu \mathrm{m}$. One can see that a fine mesh at the grain boundary is needed to sufficiently resolve the stress concentrations. These are due to different crystallographic orientations of the grains. Only the smallest required element size is approximately small enough for the selected $R$. On macro level, insufficiently small element size can result in almost an order of magnitude different stress response, see Fig. 8. However, uniformly reducing the element size results in an enormous total number of finite elements. To mitigate this, gradient mesh density should be employed, with dense mesh at the grain boundaries and coarser mesh towards the center of the grain, see [13]. In our 212 grain cases the coarse mesh in the center of a grain was obtained by inserting a circular partition and using exactly 4 elements along its circumference.
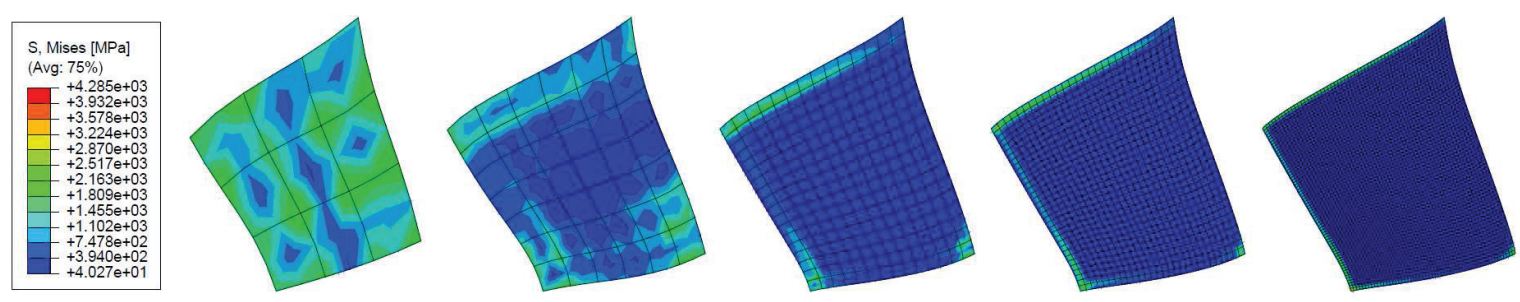

FIGURE 7. Variation of $\sigma_{\text {Mises }}$ within grain 8 for different mesh densities. $R=0.5 \mu \mathrm{m}$, element size at the grain boundary is 12.5 , 5, 1.9, 1 and $0.5 \mu \mathrm{m}$ 


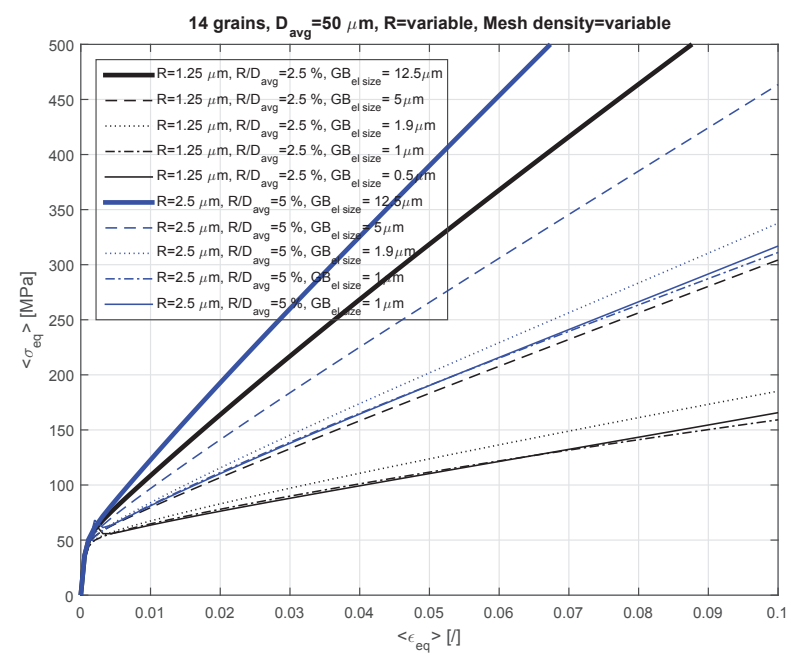

FIGURE 8. The finite element size effect on the macroscopic response of an 14 grain aggregate.

\section{CONCLUSIONS}

This work addresses the illustration of physical size effect through a strain gradient crystal plasticity model to be used in the micro-forming processes. The importance of the $R / D_{\text {avg }}$ ratio between the internal length scale and the average grain size is studied. Identical microscopic and macroscopic behavior of a polycrystalline aggregate is obtained for the same $R / D_{\text {avg }}$ ratios. The larger the ratio the stiffer the macroscopic response is and it also affects the proof stress. It is concluded that the finite element size should be strictly controlled around the high stress concentration areas where it should be smaller than the $R$ value. The element size should be preferably less than $R / 2$ to obtain sufficient resolution and convergence of the local and global responses of the aggregate. For this, a suitable gradient-type finite element mesh is required so that the model size is still manageable. The current study does not consider a grain boundary model taking into account physical plastic slip transmission and accumulation phenomena (see [14]) and the statistical size effect which are the scope of the extended version of this work.

\section{ACKNOWLEDGMENTS}

Tuncay Yalçinkaya gratefully acknowledges the support by the Scientific and Technological Research Council of Turkey (TÜBİTAK) under the 3001 Programme (Grant No. 215M381).

\section{REFERENCES}

[1] T. Yalcinkaya, W. Brekelmans, and M. Geers, J. Mech. Phys. Solids 59, 1-17 (2011).

[2] T. Yalçinkaya, W. Brekelmans, and M. Geers, Int. J. Solids Struct. 49, 2625-2636 (2012).

[3] F. Aurenhammer, ACM Computing Surveys 23, 345-405 (1991).

[4] A. Di Schino and J. Kenny, Mater. Lett. 57, 3182-3185 (2003).

[5] X. Feaugas and H. Haddou, Metall. Mater. Trans. A 34A, 2329-2340 (2003).

[6] C. Bayley, W. Brekelmans, and M. Geers, Int. J. Solids Struct. 43, 7268-7286 (2006).

[7] W. Nix and H. Gao, J. Mech. Phys. Solids 46, 411-425 (1998).

[8] K. Aifantis, J. Senger, D. Weygand, and M. Zaiser, Materials Science and Engineering 3 (2009).

[9] G. Voyiadjis and R. Abu Al-Rub, Int. J. Solids Struct. 42, 3998-4029 (2005).

[10] E. Hall, Proceedings of the Physical Society. Section B 64, p. 747 (1951).

[11] N. Petch, Journal of the Iron and Steel Institute 174, 25-28 (1953).

[12] L. E. Murr, Interfacial Phenomena in Metal and Alloys (Addison-Wesley Publishing Company, 1975).

[13] C. Dahlberg and J. Faleskog, Eur. J Mech. A-Solid 44, 1-16 (2014).

[14] I. Ozdemir and T. Yalçinkaya, Comput. Mech. 54, 255-268 (2014). 